\title{
Highlighting the burden of malarial infection and disease in the neonatal period: making sense of different concepts
}

\author{
Abel Nhama ${ }^{1,2}$, Rosauro Varo ${ }^{1,3}$ and Quique Bassat ${ }^{1,3,4,5,6,7^{*}}$ (])
}

\section{Background}

In malaria-endemic areas, the first year of life is indisputably considered one of the most vulnerable periods for malaria disease, and malaria-associated mortality in this age group is typically high, usually as a consequence of severe anaemia, among other possible complications [1]. However, malaria disease during the first 4 weeks of life (which includes both congenital and neonatal malaria) is comparatively much less common than among older infants. Congenital and neonatal malaria show important differences in terms of acquisition route and timing of the infection. They both are also importantly influenced by background malaria endemicity and degrees of population-acquired immunity.

\section{Acquisition route}

Malaria in the first weeks of life can essentially arise from two very distinct transmission mechanisms. It can either be vertically transmitted from an infected pregnant mother to the child (in utero or during delivery) [2], or it can be acquired through a mosquito bite transmitting the infective forms of the parasite (sporozoite). In the first case, it is often challenging to determine whether the infection is derived from parasites acquired before delivery or as a result of contamination by maternal blood at birth. In the second case, and given the necessary incubation period of the parasite $(\sim 10-14$ days, species

*Correspondence: quique.bassat@isglobal.org

${ }^{7}$ Barcelona Institute for Global Health, Carrer Roselló 132, Barcelona, Sobreátic 08036, Spain

Full list of author information is available at the end of the article dependant), it would be difficult to conceive any newborn becoming sick during its first week of life through this transmission route. By convention, therefore, it has been agreed to use different nomenclature to differentiate the assumed transmission route. Malaria cases detected in the first 7 days of life are called congenital malaria, whereas those detected henceforth and until 28 days of life are termed neonatal malaria. Such a distinction between congenital and neonatal malaria is relevant in endemic areas, because prevention methods designed to avoid infection in the baby should differentially target the pregnant mother or the baby. Outside of malaria endemic areas, malaria in the newborn is always assumed to have been transmitted congenitally, irrespective of the time of onset of symptoms.

\section{The importance of the background immunity status of the population against malaria}

One of the fundamental factors in the current understanding of the pathophysiology and clinical presentation of malaria is related to the presence (or not) of any degree of acquired immunity to malaria from the human host [3]. Indeed, in malaria-endemic areas, repeated exposure to infectious mosquito bites leads to the progressive development of a natural immune response that will eventually protect children and adults from the deleterious consequences of malaria disease (including death). However, such acquired immunity is not sterilizing, and therefore, infections may still occur throughout life, even though they are not necessarily accompanied by clinical disease, a phenomenon known as malaria tolerance [4]. Thus, in malaria-endemic settings with moderate to high

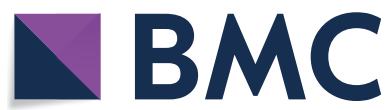

(c) The Author(s) 2020. This article is licensed under a Creative Commons Attribution 4.0 International License, which permits use, sharing, adaptation, distribution and reproduction in any medium or format, as long as you give appropriate credit to the original author(s) and the source, provide a link to the Creative Commons licence, and indicate if changes were made. The images or other third party material in this article are included in the article's Creative Commons licence, unless indicated otherwise in a credit line to the material. If material is not included in the article's Creative Commons licence and your intended use is not permitted by statutory regulation or exceeds the permitted use, you will need to obtain permission directly from the copyright holder. To view a copy of this licence, visit http://creativecommons.org/licenses/by/4.0/. The Creative Commons Public Domain Dedication waiver (http://creativecommons.org/publicdomain/zero/1.0/) applies to the data made available in this article, unless otherwise stated in a credit line to the data. 
transmission, it is very common to find malaria parasites among symptomless individuals, and such infections may not necessarily require treatment as they can be considered incidental findings. This is radically different to what occurs in settings where malaria is less common or where autochthonous transmission does not occur, as immunity will not have developed, and all individuals remain equally naïve and susceptible to the disease once they get infected, irrespective of their age. Newborns could also be considered similarly naïve and susceptible to malaria, although they may passively acquire protecting antibodies from their mothers during pregnancy and/or breastfeeding.

\section{The perceived scarce malaria burden during the neonatal period}

As a demonstrative example, an analysis of morbidity surveillance data from outpatients attending a Mozambican district hospital in a high transmission setting, and for which all patients with fever were systematically screened for malaria, signalled that only $2.7 \%$ of all newborns seen $(\sim 2000)$ had a clinical diagnosis of malaria, whereas this percentage increased to up to $40 \%$ among older children [5]. Several factors could explain why malaria during the neonatal period has been traditionally considered a rare phenomenon in malaria-endemic areas. As previously mentioned, in these settings, the passive transfer of antibodies from pregnant mothers, which generally start to wane after the first 3 months of life would explain why clinical malaria is uncommon before that period. Fetal haemoglobin $(\mathrm{HbF})$, whose presence inside the young infant's erythrocytes is known to hinder parasite growth, may also contribute to reduced vulnerability in the newborn to malaria, during the time it can still be detected in circulation [6]. Finally, the "sheltering" conferred during the first month of life to the many layers of clothing that newborns usually are dressed with, together with the closeness to the mother (for instance sleeping together under the same bed net), cannot be disregarded, as additional factors of protection. Of these arguments, the first two also explain why congenital malaria is often clinically silent and why potential symptoms among those cases are either absent, or rather unspecific (including fever or hypothermia, jaundice, hepato-splenomegaly, poor feeding) and often mild in nature. However, there is an evident discrepancy between clinical malaria cases in this period (relatively infrequent), and the prevalence of malaria infections (much more common), when proactively investigated. Indeed, when using more sensitive diagnostic methods, congenital infections seem to occur quite frequently among neonates born of women experiencing malaria during pregnancy, as confirmed by the high prevalence (up to $32 \%$ in some series) of PCRdetected malarial infection in cord blood [7].

\section{Clinical malaria among newborns of naïve to malaria pregnant mothers}

The aforementioned scenario from malaria-endemic areas cannot be extrapolated to babies born of malarianaïve-or with little acquired malaria immunity-pregnant women, whereby congenital transmission usually leads to a clinically overt and life-threatening episode. This is the situation of women infected with malaria in low-endemic settings, or as travellers. Given this, it is no surprise that the literature on congenital malaria is very much biased towards case-reports/series from very low- or nonendemic areas [8-13], whereby the differential diagnosis of the sick newborn typically requires excluding bacterial sepsis. Needless to say, these are often medical emergencies, with the added complexity of being tortuous to diagnose when expertise in malaria is missing and malaria is not initially considered as part of the differential diagnosis.

\section{A new approximation to the real burden of congenital and neonatal malaria}

Celestin Danwang and colleagues [14] have conducted a systematic review and meta-analysis of 22 observational studies reporting on congenital (0-7 days) and neonatal (0-28 days) clinical malaria, including a total of 28,083 neonates from 14 malaria-endemic countries, in order to assess its pooled prevalence in this specific age group. Importantly, their results suggest a prevalence of congenital malaria of 40.4/1000, and a prevalence of neonatal malaria of $12 / 1000$, with no apparent differences between point estimates from pooled studies from sub-Saharan Africa, and studies from other continents, although the limited examined data from the latter forcefully limit the generalizability of their conclusions. Such results complement a similar and recently published meta-analysis [15], restricted to congenital malaria only, and assessing 8148 newborns, who postulated an overall global prevalence of $6.9 \%$, with a high inter-country variability (with estimates as high as $46.7 \%$ in Nigeria). Interestingly, the authors also confirmed congenital malaria to be more frequent in settings with unstable malaria transmission, a finding in line with the hypothesis of the importance of the immunity background in the risk of congenital malaria.

A major limitation of those two very similar metaanalyses, that can be traced back to the original studies pooled, and a major caveat for their adequate interpretation, are the difficulties in linking malaria infection to clinical disease in this particular age group. As previously mentioned, in malaria-endemic areas, attributing to malaria the causality of the symptoms seen in a 
sick newborn requires a comprehensive and thorough exclusion of other possible causes, otherwise clinical misattribution may be significant. A second important limitation is the major focus on a single continent (Africa) and species (P. falciparum), whereby very few studies (and data) have been included from other species and malaria-endemic regions of the world. Having said so, meta-analyses like the ones recently published can address the neglect and broaden the poor visibility that congenital and neonatal malaria have traditionally suffered, on account of their until recently assumed low burden of disease. They can also propose contextual frameworks based on the endemicity characteristics that can help stratify regions and countries in terms of potential risks to health of vertical transmission, thus defining more proactive protocols for malaria investigation in the sick newborn. Indeed, having succeeded on bypassing this first visibility hurdle, it is now time to focus on re-defining what particular strategies are best suited for the prevention and treatment of congenital and neonatal malaria. In this respect, approaches will need to focus both on pregnant mothers (including early diagnosis and prompt treatment, maternal chemoprevention or vaccination) and the newborn, where newborn-friendly formulations and weight-specific adjustments of the dosing of the drugs available to cure malaria will be required, in an effort to resolve some of the unmet needs [16] of this particularly vulnerable-in its own way-group.

\section{Acknowledgements}

ISGlobal acknowledges the support from the Spanish Ministry of Science and Innovation through the "Centro de Excelencia Severo Ochoa 2019-2023" Program (CEX2018-000806-S), and support from the Generalitat de Catalunya through the CERCA Program. CISM is supported by the Government of Mozambique and the Spanish Agency for International Development (AECID).

\section{Authors' contributions}

AN, RV and QB conceptualized the content and the layout of this commentary. AN and QB drafter the first version of this manuscript. All authors critically reviewed and commented all versions of this manuscript. All authors read and approved the final manuscript.

\section{Funding}

No funding was used to write this manuscript

Availability of data and materials

Not applicable.

Ethics approval and consent to participate

Not applicable.

\section{Consent for publication}

Not applicable.

\section{Competing interests}

Authors declare no competing interests.

\begin{abstract}
Author details
${ }^{1}$ Centro de Investigação em Saúde de Manhiça (CISM), Maputo, Mozambique. ${ }^{2}$ Instituto Nacional de Saúde (INS), Maputo, Mozambique. ${ }^{3}$ ISGlobal, Hospital Clínic, Universitat de Barcelona, Barcelona, Spain. ${ }^{4}$ ICREA, Pg. Lluís Companys 23, Barcelona 08010, Spain. ${ }^{5}$ Pediatric Infectious Diseases Unit, Pediatrics Department, Hospital Sant Joan de Déu (University of Barcelona), Barcelona, Spain. ${ }^{6}$ Consorcio de Investigación Biomédica en Red de Epidemiología y Salud Pública (CIBERESP), Madrid, Spain. ${ }^{7}$ Barcelona Institute for Global Health, Carrer Roselló 132, Barcelona, Sobreátic 08036, Spain.
\end{abstract}

Received: 17 August 2020 Accepted: 25 August 2020

Published online: 28 August 2020

References

1. WHO. World malaria report 2019. Geneva, World Health Organization, 2019.

2. Menendez C, Mayor A. Congenital malaria: the least known consequence of malaria in pregnancy. Semin Fetal Neonatal Med. 2007;12:207-13.

3. Doolan DL, Dobaño C, Baird JK. Acquired immunity to malaria. Clin Microbiol Rev. 2009;22:13-36.

4. Galatas B, Bassat Q, Mayor A. Malaria parasites in the asymptomatic: looking for the hay in the haystack. Trends Parasitol. 2016;32:296-308.

5. Guinovart C, Bassat Q, Sigaúque B, Aide P, Sacarlal J, Nhampossa T, et al. Malaria in rural Mozambique. Part I children attending the outpatient clinic. Malar J. 2008;7:36.

6. Amaratunga C, Lopera-Mesa TM, Brittain NJ, Cholera R, Arie T, Fujioka $\mathrm{H}$, et al. A role for fetal hemoglobin and maternal immune lgG in infant resistance to Plasmodium falciparum malaria. PLoS ONE. 2011;6:e14798.

7. Tobian AA, Mehlotra RK, Malhotra I, Wamachi A, Mungai P, Koech D, et al. Frequent umbilical cord-blood and maternal-blood infections with Plasmodium falciparum, P malariae, and P. ovale in Kenya. J Infect Dis. 2000;182:558-63.

8. McGovern LM, Boyce TG, Fischer PR. Congenital infections associated with international travel during pregnancy. J Travel Med. 2007;14:117-28.

9. Rai P, Majumdar K, Sharma S, Chauhan R, Chandra J. Congenital malaria in a neonate: case report with a comprehensive review on differential diagnosis, treatment and prevention in Indian perspective. J Parasit Dis. 2015;39:345-8.

10. Tao ZY, Fang Q, Liu X, Culleton $R$, Tao L, Xia H, et al. Congenital malaria in China. PLoS Negl Trop Dis. 2014;8:e2622.

11. Vottier G, Arsac M, Farnoux C, Mariani-Kurkdjian P, Baud O, Aujard Y. Congenital malaria in neonates: two case reports and review of the literature. Acta Paediatr. 2008;97:505-8.

12. Lesko CR, Arguin PM, Newman RD. Congenital malaria in the United States: a review of cases from 1966 to 2005. Arch Pediatr Adolesc Med. 2007;161:1062-7.

13. Pengsaa K. Congenital malaria in Thailand. Ann Trop Paediatr. 2007;27:133-9.

14. Danwang C, Bigma JJ, Tuku Nzalie RN, Robert A. Epidemiology of clinical congenital and neonatal malaria in endemic settings: a systematic review and meta-analysis. Mala J. 2020;19:in press.

15. Bilal JA, Malik EE, Al-Nafeesah A, Adam I. Global prevalence of congenital malaria: A systematic review and meta-analysis. Eur J Obstet Gynecol Reprod Biol. 2020;S0301-2115(20)30392-4. Online ahead of print.

16. D'Alessandro U, Ubben D, Hamed K, Ceesay SJ, Okebe J, Taal M, et al. Malaria in infants aged less than six months -is it an area of unmet medical need? Malar J. 2012;11:400.

\section{Publisher's Note}

Springer Nature remains neutral with regard to jurisdictional claims in published maps and institutional affiliations. 